\title{
FUZZY NEIGHBORHOOD STRUCTURES ON PARTIALLY ORDERED GROUPS
}

\author{
KAMEL EL-SAADY and M. Y. BAKIER
}

Received 5 May 2001

\begin{abstract}
Ahsanullah (1988) showed the compatibility between group structures and $I$-fuzzy neighborhood systems. In this paper, we require not only that the $I$-fuzzy neighborhood systems be compatible with the group structures, but also compatible with the order relation, in one sense or another.
\end{abstract}

2000 Mathematics Subject Classification: 54A40, 54H15, 54E15, 06F15, 20F60, $22 \mathrm{~A} 05$.

1. Introductions. In [8], Katsaras combine the concepts of [0,1]-topology and order structure to bring out the so-called ordered fuzzy topological spaces. Several authors have continued on the work of Katsaras in the area of $[0,1]$-topology and order [3, 4, $10]$.

In [2] Ahsanullah introduced the notion of $I$-fuzzy neighborhood groups. In this paper, we aim to introduce and study the concept of $I$-fuzzy neighborhood structures on ordered groups.

2. Preliminaries. Let $X$ be a nonempty set. A relation $\leq$ on $X$ is said to be preorder if it is reflexive and transitive. An antisymmetric preorder is said to be a partially order. By a preordered (resp., an ordered) set, we mean a set $X$ with a preorder (resp., a partially order) relation on it and we denote it by $(X, \leq)$. Every set can be considered as a partially ordered set equipped with the discrete order $(x \leq y$ if and only if $x=y$ ).

A function $f$ from a preordered set $(X, \leq)$ to a preordered set $\left(X^{\prime}, \leq^{\prime}\right)$ is called isotone or order-preserving (resp., antitone or order-inverting) if $x \leq y$ in $X$ implies $f(x) \leq^{\prime} f(y)$ (resp., $\left.f(y) \leq^{\prime} f(x)\right)$ in $X^{\prime}$. The function $f$ is said to be order isomorphism if it is bijection and $(\forall x, y \in X) x \leq y \Leftrightarrow f(x) \leq^{\prime} f(y)$.

Suppose that $(G, *)$ is a semigroup and that $G$ is endowed with an order $\leq$. We say that $(G, *, \leq)$ is an ordered semigroup if the low of composition and the order are related by the property: for all $x, y \in G$

$$
x \leq y \Longrightarrow(\forall z \in G) x * z \leq y * z, \quad z * x \leq z * y .
$$

If $\left(G_{1}, T_{1}, \leq_{1}\right)$ and $\left(G_{2}, T_{2}, \leq_{2}\right)$ are ordered semigroups. A mapping $f: G_{1} \rightarrow G_{2}$ is said to be order-homomorphism if it is both isotone and semigroup homomorphism. By an ordered group we mean an ordered semigroup which is a group.

In this paper, we use the multiplicative ordered group $(G, \cdot, \leq)$ which is sometimes written as $(G, \leq)$. 
Combining the notion of order-isomorphism and group isomorphism, we say that an ordered group $\left(G_{1}, \leq_{1}\right)$ is OG-isomorphic to an ordered group $\left(G_{2}, \leq_{2}\right)$ if there is a mapping $f: G_{1} \rightarrow G_{2}$ which is both order isomorphism and group isomorphism.

An $I$-fuzzy set $\mu$, in a preordered set $(X, \leq)$, is called increasing (resp., decreasing) if $x \leq y$ implies $\mu(x) \leq \mu(y)$ (resp., $\mu(y) \leq \mu(x))$ [8].

A Chang-Goguen $L$-topology (cf. [5, 6, 7]) on a set $X$ is a subset $\tau \subset L^{X}$, closed under finite infs and arbitrary sups. A pair $(X, \tau)$ is called a Chang-Goguen $L$-topological space; $(X, \tau)$ is called stratified $L$-topological space if $\tau$ contains all the constant $L$-fuzzy sets. The category of Chang-Goguen $L$-topological spaces (resp., stratified Chang-Goguen $L$-topological spaces) is denoted by $\mid L$-Top $\mid$ (resp., $\mid S L$-Top $\mid$ ). Both $\mid L$-Top $\mid$ and $\mid S L$-Top $\mid$ are topological categories. If $L=I=[0,1]$, the above categories are denoted by $\mid I$-Top $\mid$ and $\mid S I$-Top $\mid$, respectively.

By an $I$-topological (resp., stratified $I$-topological) ordered space are we mean a triplet $(X, \leq, \tau)$, consisting of a partially ordered set $(X, \leq)$ and an $I$-topology (resp., stratified $I$-topology) $\tau$ on $X$.

By $\mid I$-TopOS| (resp., $\mid S I$-TopOS $\mid$ ), we mean the category of all $I$-topological (resp., stratified $I$-topological) ordered spaces as object and all order-preserving continuous mappings between them as morphisms.

The order $\leq$, in an $I$-topological ordered space $(X, \leq, \tau)$, is said to be closed [8] if and only if the following condition holds: if $x \nless y$, then there are neighborhoods $\mu$, $\rho$ of $x, y$, respectively, such that $i(\mu) \wedge d(\rho)=0$.

Let $(X, \leq, \tau)$ be an $L$-topological ordered space. If the order is closed, then $X$ is Hausdorff [8].

An $I$-fuzzy quasi-uniformity [9] is a subset $\mathbf{U}$ of $I^{X \times X}$ which is prefilter and has the following three properties:

(1) $\alpha(x, x)=1 \forall \alpha \in \mathbf{U}$ and $\forall x \in X$,

(2) $\forall \alpha \in \mathbf{U}, \forall \varepsilon>0, \exists \alpha_{1} \in \mathbf{U}$ such that $\alpha_{1} \circ \alpha_{1}-\varepsilon \leq \alpha$,

(3) $\mathbf{U}=\mathbf{U}$, that is, for every family $\left\{\alpha_{\varepsilon} \in \mathbf{U}, \varepsilon \in I_{0}\right\}$ we have $\sup _{\varepsilon \in I\left(\alpha_{\varepsilon}-\varepsilon\right) \in \mathbf{U}}$.

The family $\mathbf{U}^{-1}=\left\{\alpha^{-1}: \alpha \in \mathbf{U}, \alpha^{-1}(x, y)=\alpha(y, x)\right\}$ is an $I$-fuzzy quasi-uniformity on $X$ called the conjugate of $\mathbf{U}$. We denote by $\mathbf{U}^{*}$ the $I$-fuzzy uniformity which generated by $\mathbf{U}$, that is, $\mathbf{U}^{*}=\mathbf{U} \vee \mathbf{U}^{-1}=\left\{\alpha \wedge \alpha^{-1}: \alpha \in \mathbf{U}, \alpha^{-1} \in \mathbf{U}^{-1}\right\}$. The $I$-fuzzy quasiuniformity $\mathbf{U}$ can generate an order, say $\leq_{u}$, by setting

$$
x \leq_{u} y \Longleftrightarrow \begin{cases}\alpha(x, z) \leq \alpha(y, z) & \forall z \geq x, y, \\ \alpha(x, z) \geq \alpha(y, z) & \forall z \leq x, y .\end{cases}
$$

A triplet $\left(X, \leq, \mathbf{U}^{*}\right)$, consisting of an ordered set $(X, \leq)$ and an $I$-fuzzy uniformity $\mathbf{U}^{*}$, is called an $I$-fuzzy uniform ordered space [10] if there exists an $I$-fuzzy quasiuniformity $\mathbf{U}$ on $X$ such that $\mathbf{U}^{*}=\mathbf{U} \vee \mathbf{U}^{-1}$ and $G(\leq)=G\left(\leq_{u}\right)$.

DEFINITION 2.1 [10]. Let $\left(X_{1}, \mathbf{U}_{1}\right)$ and $\left(X_{2}, \mathbf{U}_{2}\right)$ be $I$-fuzzy quasi-uniform spaces. A mapping $f:\left(X_{1}, \mathbf{U}_{1}\right) \rightarrow\left(X_{2}, \mathbf{U}_{2}\right)$ is said to be quasi-uniformly continuous if and only if $\forall \alpha_{2} \in \mathbf{U}_{2}, \exists \alpha_{1} \in \mathbf{U}_{1}$ such that $\alpha_{1} \in(f \times f)^{-1}\left(\alpha_{2}\right)$. Where $f$ is called quasi-uniform equivalence if $f$ is bijective and both $f$ and $f^{-1}$ are quasi-uniformly continuous. 
DefinITION 2.2 [10]. A mapping $f:\left(X, \leq, \mathbf{U}^{*}\right) \rightarrow\left(X_{1}, \leq_{1}, \mathbf{U}^{*}\right)$ is said to be uniformly order-mapping if there exist $I$-fuzzy quasi-uniformities $u$ and $u_{1}$ on $X$ and $X_{1}$, respectively such that

(i) $\mathbf{U}^{*}=\mathbf{U} \vee \mathbf{U}^{-1}$ and $G(\leq)=G\left(\leq_{u}\right)$;

(ii) $\mathbf{U}_{1}^{*}=\mathbf{U}_{1} \vee \mathbf{U}_{1}^{-1}$ and $G\left(\leq_{1}\right)=G\left(\leq_{u_{1}}\right)$;

(iii) $f:(X, \mathbf{U}) \rightarrow\left(X_{1}, \mathbf{U}_{1}\right)$ is quasi-uniformly continuous.

Definition 2.3 [2]. Let $(G, \cdot)$ be a group and let $\boldsymbol{\kappa}$ be an $I$-fuzzy neighborhood system on $G$. Then, the triplet $(G, \cdot, t(\mathrm{~N}))$ is called $I$-fuzzy neighborhood group if and only if the following conditions are fulfilled:

(1) the mapping $m:(G \times G, t(\mathrm{\kappa}) \times t(\mathrm{\kappa})) \rightarrow(G, t(\mathrm{\kappa})):(x, y) \rightarrow x y$ is continuous;

(2) the mapping $r:(G, t(\mathrm{~N})) \rightarrow(G, t(\mathrm{~N})): x \rightarrow x^{-1}$ is continuous.

Proposition 2.4 [2]. Let $(G, \cdot)$ be a group and let $\mathrm{N}$ be an I-fuzzy neighborhood system on $G$. Then, $(G, \cdot, t(\mathrm{~N}))$ is an I-fuzzy neighborhood group if and only if the mapping

$$
h:(G \times G, t(\kappa) \times t(\kappa)) \longrightarrow(G, t(\kappa)):(x, y) \longrightarrow x y^{-1}
$$

is continuous

\section{Fuzzy neighborhood ordered groups}

DEFINITION 3.1. A triplet $(G, \leq, t(\mathrm{~K}))$ is called $I$-fuzzy neighborhood ordered groups if the following statements hold:

(1) $(G, \leq)$ is a partially ordered group;

(2) $(G, t(\aleph))$ is an $I$-fuzzy neighborhood group;

(3) the order $\leq$ is closed.

By $\mid I$-FNOGr $\mid$, we mean the category of all $I$-fuzzy neighborhood ordered groups as objects and all order-preserving homeomorphisms between them as morphisms.

In agreement with [1], a faithful functor $T: A \rightarrow$ Set is said to be topological (monotopological) if and only if, given any index class $\left(\left(X_{j}, \xi_{j}\right): j \in J\right)$ of $A$-objects indexed by a class $J$ and any source (resp., mono-source) $\left(f_{j}: X \rightarrow X_{j}\right)$ in Set, there exists a unique $A$-structure $\xi$ on $X$ which is initial with respect to $\left(f_{j}: X \rightarrow\left(X_{j}, \xi_{j}\right)\right)_{j \in J}$, that is, such that for any $A$-object $(Y, \zeta)$, a mapping $h:(Y, \zeta) \rightarrow(X, \xi)$ is an $A$-morphism if and only if for every $j \in J$, the composition $f_{j} \circ h:(Y, \zeta) \rightarrow\left(X_{j}, \xi_{j}\right)$ is an $A$-morphism. Also, we have that the constant function lift to morphism in $A$ and the $A$-fibre $T^{-1}(S)$ for any set $S$ is small.

Proposition 3.2. The category $|I-\mathrm{FNOGr}|$ is mono-topological.

Proof. The forgetful functor $T:|I-\mathrm{FNOGr}| \rightarrow \mid$ Group $\mid$ is given by $T(G, \leq, t(\mathrm{\aleph}))=$ $G$. For some index class $J$, let $\left(G_{\alpha}, \leq_{\alpha}, t\left(\aleph_{\alpha}\right)\right) \in \mid I-$ FNOGr $\mid$ and $\left(f_{\alpha}: G \rightarrow G_{\alpha}\right)_{\alpha \in J}$ be a monosource in $\mid$ Group $\mid$. Let $\times$ be the $I$-fuzzy neighborhood system making the monosource

$$
\left(f_{\alpha}:(G, t(\kappa)) \longrightarrow\left(G_{\alpha}, t\left(\aleph_{\alpha}\right)\right)\right)_{\alpha \in J}
$$


initial and let $\leq$ be the order defined by $x \leq y$ if and only if $f_{\alpha}(x) \leq_{\alpha} f_{\alpha}(y)$ for all $\alpha \in J$. Then $(G, \leq, t(\mathrm{~N})) \in \mid I-$ FNOGr $\mid$. Initiality of the mono-source

$$
\left(f_{\alpha}:(G, \leq, t(\kappa)) \longrightarrow\left(G_{\alpha}, \leq_{\alpha}, t\left(\aleph_{\alpha}\right)\right)\right)_{\alpha \in J}
$$

can easily be checked; thus $T$ is mono-topological. The other conditions for a monotopological category are clearly met.

Proposition 3.3. Let $(G, \leq, t(\mathrm{\kappa})) \in|I-\mathrm{FNOGr}|$. Then, for $x, a \in G$,

(i) the mapping $L_{a}: G \rightarrow G$ (resp., $R_{a}: G \rightarrow G$ ) defined by $x \rightarrow a x$ (resp., $x \rightarrow x a$ ) is an order-preserving homeomorphism;

(ii) the mapping $r:(G, t(\mathrm{~N})) \rightarrow(G, t(\mathrm{~N})): x \rightarrow x^{-1}$ is an order-inverting homeomorphism.

Proof. The proof follows from Definition 2.3.

LEMMA 3.4. Let $(G, \leq, t(\mathrm{\kappa})) \in|I-\mathrm{FNOGr}|$ and $\mu$ be an increasing (resp., decreasing) I-fuzzy set in $G$, then

(i) $R_{a}^{-1}(\mu)$ is increasing (resp., decreasing);

(ii) $r^{-1}(\mu)$ is decreasing (resp., increasing).

Proof. Let $\mu$ be an increasing $I$-fuzzy set.

(i) We have

$$
\begin{aligned}
& R_{a}^{-1}(\mu)(x)=\mu\left(R_{a}(x)\right)=\mu(x a) \leq \mu(y a)=\mu\left(R_{a}(y)\right), \\
& R_{a}^{-1}(\mu)(x)=\mu\left(R_{a}(x)\right) \leq \mu\left(R_{a}(y)\right)=R_{a}^{-1}(\mu)(y),
\end{aligned}
$$

that is, $R_{a}^{-1}(\mu)(x) \leq R_{a}^{-1}(\mu)(y)$ whenever $x \leq y$.

(ii) The mapping $r: G \rightarrow G$ is decreasing, then

$$
r^{-1}(\mu)(x)=\mu(r(x))=\mu\left(x^{-1}\right) \geq \mu\left(y^{-1}\right)=\mu(r(y))=r^{-1}(\mu)(y),
$$

that is, $r^{-1}(\mu)$ is decreasing.

Proposition 3.5. If $(G, \leq, t(\mathrm{\kappa})) \in|I-\mathrm{FNOGr}|$ and $\mu$ is an increasing (resp., decreasing) open I-fuzzy set in $G$ and $\rho \in I^{G}$, then the I-fuzzy set $(\mu \cdot \rho)$ is an increasing (resp., decreasing) open I-fuzzy set in $G$.

Proof. By [2, Proposition 1.10], an $I$-fuzzy set $(\mu \cdot \rho)$ is open. To prove the second part, let $x, y \in G$ with $x \leq y$ and $\mu$ be increasing $I$-fuzzy, then

$$
\begin{aligned}
\mu \cdot \rho(x) & =\sup _{x=s \cdot t} \mu(s) \wedge \rho(t)=\sup _{t \in G} \mu\left(x t^{-1}\right) \wedge \rho(t) \\
& =\sup _{t \in G} \mu\left(R_{t}^{-1}(x)\right) \wedge \rho(t)=\sup _{t \in G} R_{t}(\mu)(x) \wedge \rho(t) .
\end{aligned}
$$

But the mapping $R_{t}: G \rightarrow G: x \rightarrow x t$ is increasing, then, by fixing $t \in G$, it follows that

$$
\mu \cdot \rho(x)=\sup _{t \in G} R_{t}(\mu)(x) \wedge \rho(t) \leq \sup _{t \in G} R_{t}(\mu)(y) \wedge \rho(t)=\mu \cdot \rho(y),
$$

that is, $I$-fuzzy set $\mu \cdot \rho$ is increasing. 
Proposition 3.6. Let $(G, \leq, t(\mathrm{\aleph})) \in|I-\mathrm{FNOGr}|$, then for all increasing (resp., decreasing) I-fuzzy set $\mu \in \mathrm{N}(e)$ and for all $\varepsilon \in I_{0}$, there exists $\rho \in \mathrm{\kappa}(e)$ such that $i(\rho \cdot \rho)-\varepsilon \leq \mu$ (resp., $d(\rho \cdot \rho)-\varepsilon \leq \mu)$.

Proof. Since $(G, t(\kappa))$ is an $I$-fuzzy neighborhood group, then the continuity of the mapping $m:(G \times G, t(\kappa) \times t(\kappa)) \rightarrow(G, t(\kappa)):(x, y) \rightarrow x y$ is equivalent to the fact that $\forall \mu \in \mathrm{N}(e)$ and $\forall \varepsilon \in I_{0}$, there exists $\rho \in \mathrm{N}(e)$ (see [2, Proposition 2.5]) such that $\rho \cdot \rho-\varepsilon \leq \mu$. If we choose $\mu$ to be increasing then

$$
\rho \cdot \rho \leq i(\rho \cdot \rho) \leq \mu+\varepsilon,
$$

where $i(\rho \cdot \rho)$ is the smallest increasing $I$-fuzzy set containing $(\rho \cdot \rho)$ and it follows that $i(\rho \cdot \rho)-\varepsilon \leq \mu$ and this completes the proof.

4. Fuzzy quasi-uniformity on $I$-fuzzy neighborhood ordered groups. As given in [2], if $(G, \cdot)$ is a group, then we define

$$
\begin{aligned}
& \mu_{L}: G \times G \longrightarrow I, \quad \text { where } \mu_{L}(x, y)=\mu\left(x^{-1} y\right), \\
& \mu_{R}: G \times G \longrightarrow I, \quad \text { where } \mu_{R}(x, y)=\mu\left(y x^{-1}\right) .
\end{aligned}
$$

If $(G, \cdot, t(\aleph))$ is an $I$-fuzzy neighborhood group and $\mu \in \aleph(e)$, then $\mu_{L}$ (resp., $\mu_{R}$ ) is called the left (resp., right) $I$-fuzzy entourages associated with $\mu$. We can easily note that the left (resp., right) $I$-fuzzy entourages $\mu_{L}$ (resp., $\mu_{R}$ ) is not symmetric, if $x \neq$ $y$, then $y^{-1} x \neq e \neq x^{-1} y$ and this implies that $\mu_{L}(x, y)=\mu\left(x^{-1} y\right) \neq \mu\left(y^{-1} x\right)=$ $\mu_{L}(y, x)$. Also, $\mu_{R}(x, y) \neq \mu_{R}(y, x)$.

In the sequel, we use $\boldsymbol{N}^{i}(e)$ (resp., $\left.\mathrm{s}^{d}(e)\right)$ to denote the system of all increasing (resp., decreasing) $I$-fuzzy neighborhoods of $e$. From the above discussion we have the following easily established result.

ThEOREM 4.1. Let $(G, \leq, t(\mathrm{~N})) \in|I-\mathrm{FNOGr}|$ and $\mathrm{\kappa}^{i}(e)$ (resp., $\left.\mathrm{\aleph}^{d}(e)\right)$ denote the system of all increasing (resp., decreasing) I-fuzzy neighborhoods of e. Then,

(i) the family $\beta_{L}$ (resp., $\left.\beta_{R}\right)=\left\{\mu_{L}\right.$ (resp., $\left.\left.\mu_{R}\right): \mu \in \mathrm{N}^{i}(e)\right\}$ is a basis for the left (resp., right) I-fuzzy quasi-uniformity $u_{L}$ (resp., $u_{R}$ ) on $G$;

(ii) the family $\beta_{L}^{-1}$ (resp., $\left.\beta_{R}^{-1}\right)=\left\{\mu_{L}^{-1}\right.$ (resp., $\left.\left.\mu_{R}^{-1}\right): \mu \in \mathrm{N}^{d}(e)\right\}$ is a basis for the conjugate left (resp., right) I-fuzzy quasi-uniformity $\mathrm{U}_{L}^{-1}$ (resp., $\mathbf{U}_{R}^{-1}$ ) on $G$;

(iii) the family $\beta_{s}=\left\{\mu_{L} \wedge \mu_{R}: \mu \in \aleph^{i}(e)\right\}$ is a basis for the two-sided I-fuzzy quasiuniformity $\left(u_{R} \vee u_{L}\right)$ on $G$.

We denote $\mathbf{U}_{L} \vee \mathbf{U}_{L}^{-1}$ (resp., $\mathbf{U}_{R} \vee \mathbf{U}_{R}^{-1}$ ) by $\mathbf{U}_{L}^{*}$ (resp., $\mathbf{U}_{R}^{*}$ ). It is clear that $\mathbf{U}_{L}^{*}$ (resp., $\mathbf{U}_{R}^{*}$ ) is an $I$-fuzzy uniformity on $G$ called the left (resp., right) $I$-fuzzy uniformity generated by $\mathbf{U}_{L}$ (resp., $\mathbf{U}_{R}$ ). Also, the two-sided $I$-fuzzy uniformity $\mathbf{U}^{*}=\mathbf{U}_{R}^{*} \vee \mathbf{U}_{L}^{*}$ can be generated by the two-sided $I$-fuzzy quasi-uniformity $\left(\mathbf{U}_{R} \vee \mathbf{U}_{L}\right)$.

It is known that the entourages of the above $I$-fuzzy quasi-uniformities can generate an order on $G$ by setting

$$
x \leq^{*} y \Longleftrightarrow\left(\forall_{Z} \in G\right) \mu_{L}(y, z) \leq \mu_{L}(x, z) .
$$

The partial order $\leq^{*}$ is said to be generated by the left $I$-fuzzy quasi-uniformity $\mathbf{U}_{L}$. 
DEFINITION 4.2. Let $G_{1}, G_{2}$ be groups and $\mathbf{U}_{2}, \mathbf{U}_{2}$ be quasi-uniformities on $G_{1}$ and $G_{2}$, respectively. A mapping $f: G_{1} \rightarrow G_{2}$ is called a quasi-uniform isomorphism if it is a quasi-uniform equivalence (see Definition 2.1) and group isomorphism.

Proposition 4.3. Let $(G, \leq, t(\mathrm{~N})) \in|I-\mathrm{FNOGr}|$ and let $\mathbf{U}_{L}$ be the associated left I-fuzzy quasi-uniformity on $G$, then

(i) $L_{x}$ (resp., $\left.R_{x}\right):\left(G, \mathbf{U}_{L}\right) \rightarrow\left(G, \mathbf{U}_{L}\right)$ is a quasi-uniform isomorphism;

(ii) $L_{x}\left(\right.$ resp., $\left.R_{x}\right):\left(G, \leq, \mathbf{U}_{L}^{*}\right) \rightarrow\left(G, \leq, \mathbf{U}_{L}^{*}\right)$ is a uniformly order isomorphism.

PROoF. (i) It follows immediately from the formulas

$$
\left(L_{x} \times L_{x}\right)^{-1}\left(\mu_{L}\right)=\mu_{L} .
$$

(ii) The existence of the associated left $I$-fuzzy quasi-uniformity $\mathbf{U}_{L}$ which generate the $I$-fuzzy uniformity $\mathbf{U}_{L}^{*}$ and the order $\leq^{*}$ with $G\left(\leq^{*}\right)=G(\leq)$ and from (i) the proof becomes clear.

Proposition 4.4. Let $(G, \leq, t(\mathrm{~N})) \in|I-\mathrm{FNOGr}|$ and $\mathbf{U}_{L}$ (resp., $\left.\mathbf{U}_{R}\right)$ be the associated left (resp., right) I-fuzzy quasi-uniformity on $G$, then

(i) the mapping $r:\left(G, \mathbf{U}_{L}\right) \rightarrow\left(G, \mathbf{U}_{R}\right)$ is a quasi-uniform isomorphism;

(ii) the mapping $r:\left(G, \leq, \mathbf{U}_{L}^{*}\right) \rightarrow\left(G, \leq, \mathbf{U}_{R}^{*}\right)$ is a uniform order-isomorphism.

Proof. (i) The mapping $r: G \rightarrow G$ is a group isomorphism. But for $\mu_{R} \in \mathbf{U}_{R}$, we have that $(r \times r)^{-1}\left(\mu_{R}\right)(x, y)=\mu_{R}(r(x), r(y))=\mu_{R}\left(X^{-1}, y^{-1}\right)=\mu\left(y^{-1} x\right)$, that is, $(r \times r)^{-1}\left(\mu_{R}\right)=\tilde{\mu}_{L}$. And this means that $r:\left(G, u_{L}\right) \rightarrow\left(G, u_{R}\right)$ is a quasi-uniform equivalence and so it is quasi-uniform isomorphism.

(ii) This can be proven by Definition 2.1 and part (i) and this completes the proof.

We omit the proof of the following easily established proposition.

Proposition 4.5. Let $(G, \leq, t(\kappa))$ and $\left(G^{\prime}, \leq^{\prime}, t\left(\kappa^{\prime}\right)\right) \in|I-\mathrm{FNOGr}|$ and let $\mathrm{U}_{L}$, $\mathbf{U}_{L}^{\prime}$ be the associated left I-fuzzy quasi-uniformities on $G$ and $G^{\prime}$, respectively. Then, the order-preserving homeomorphism $f: G \rightarrow G^{\prime}$ is uniformly order-mapping.

\section{REFERENCES}

[1] J. Adámek, H. Herrlich, and G. E. Strecker, Abstract and Concrete Categories, Pure and Applied Mathematics, John Wiley \& Sons, New York, 1990.

[2] T. M. G. Ahsanullah, On fuzzy neighborhood groups, J. Math. Anal. Appl. 130 (1988), no. 1, 237-251.

[3] A. A. Allam, S. A. Hussein, and K. El-Saady, Fuzzy syntopogenous structures and order, Fuzzy Sets and Systems 63 (1994), no. 1, 91-98.

[4] M. Y. Bakier and K. El-Saady, Fuzzy topological ordered vector spaces. I, Fuzzy Sets and Systems 54 (1993), no. 2, 213-220.

[5] C. L. Chang, Fuzzy topological spaces, J. Math. Anal. Appl. 24 (1968), 182-190.

[6] J. A. Goguen, The fuzzy Tychonoff theorem, J. Math. Anal. Appl. 43 (1973), 734-742.

[7] U. Höhle and A. P. Šostak, Axiomatic foundations of fixed-basis fuzzy topology, Mathematics of Fuzzy Sets, Fuzzy Sets Ser., vol. 3, Kluwer, Massachusetts, 1999, pp. 123272.

[8] A. K. Katsaras, Ordered fuzzy topological spaces, J. Math. Anal. Appl. 84 (1981), no. 1, 44-58. 
[9] _ _ Fuzzy neighborhood structures and fuzzy quasi-uniformities, Fuzzy Sets and Systems 29 (1989), no. 2, 187-199.

[10] A. S. Mashhour, A. A. Allam, and K. El-Saady, Fuzzy uniform structures and order, J. Fuzzy Math. 2 (1994), no. 1, 57-67.

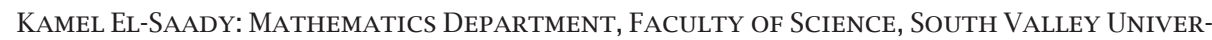
SITY, QENA 83523, EGYPT

E-mail address: e1-saady@1ycos.com

URL: http://at. yorku.ca/h/a/a/a/43.htm

M. Y. BAKIER: MATHEMAtics DePARTMENT, FACUlty of SCIEnCE, Assiut UniVersity, Assiut, EGYPT 


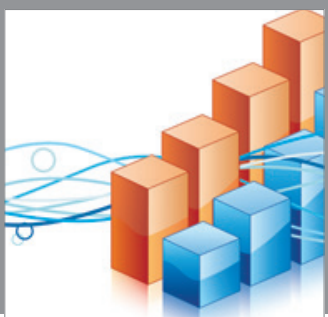

Advances in

Operations Research

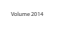

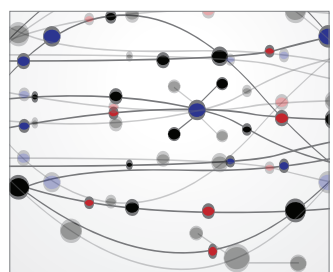

\section{The Scientific} World Journal
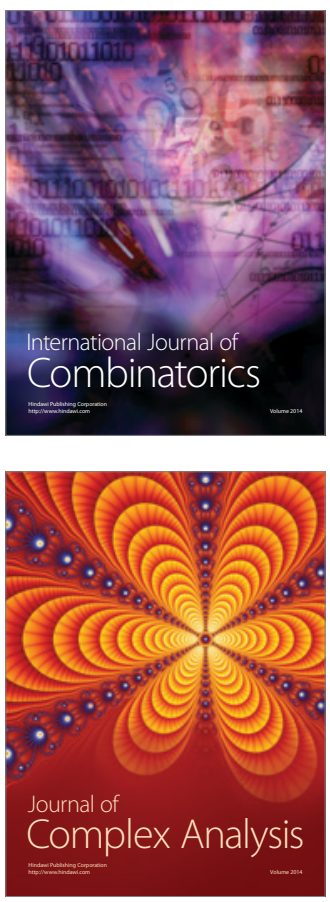

International Journal of

Mathematics and

Mathematical

Sciences
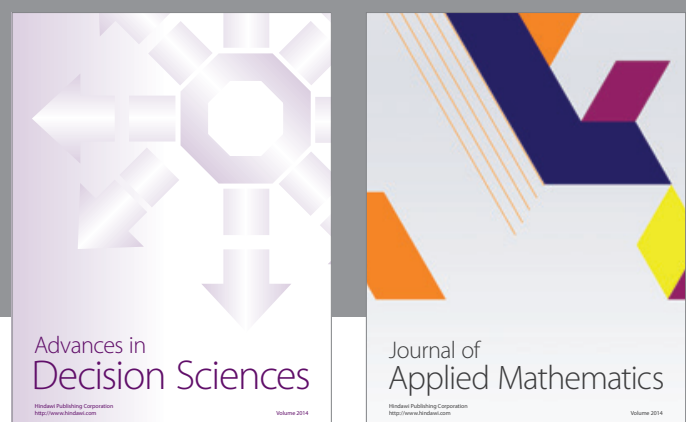

Journal of

Applied Mathematics
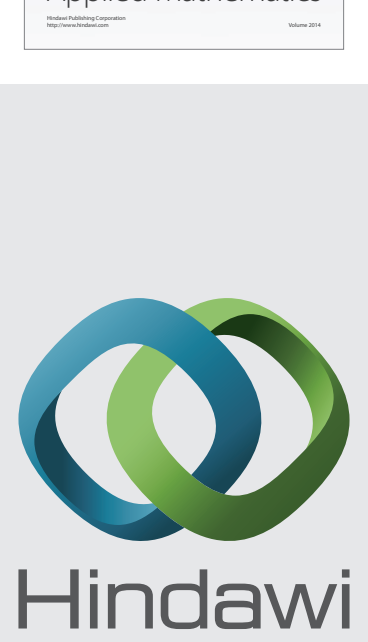

Submit your manuscripts at http://www.hindawi.com
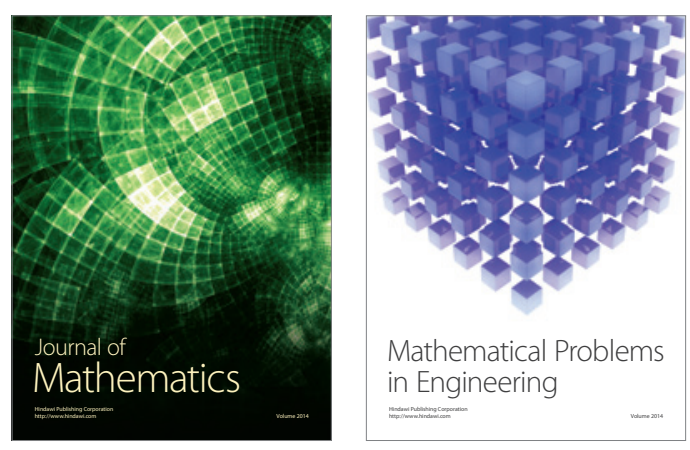

Mathematical Problems in Engineering
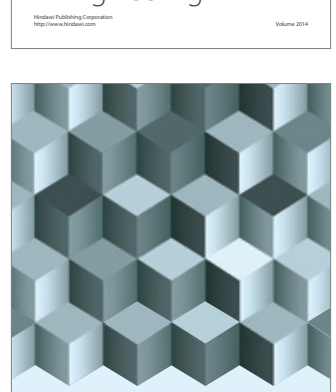

Journal of

Function Spaces
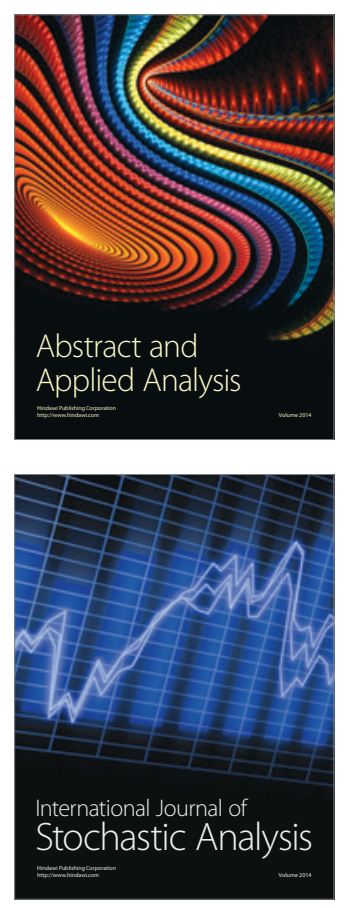

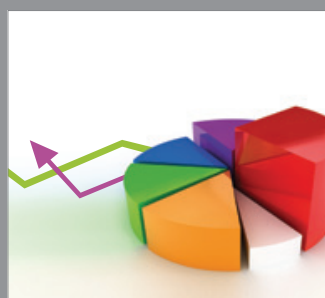

ournal of

Probability and Statistics

Promensencen
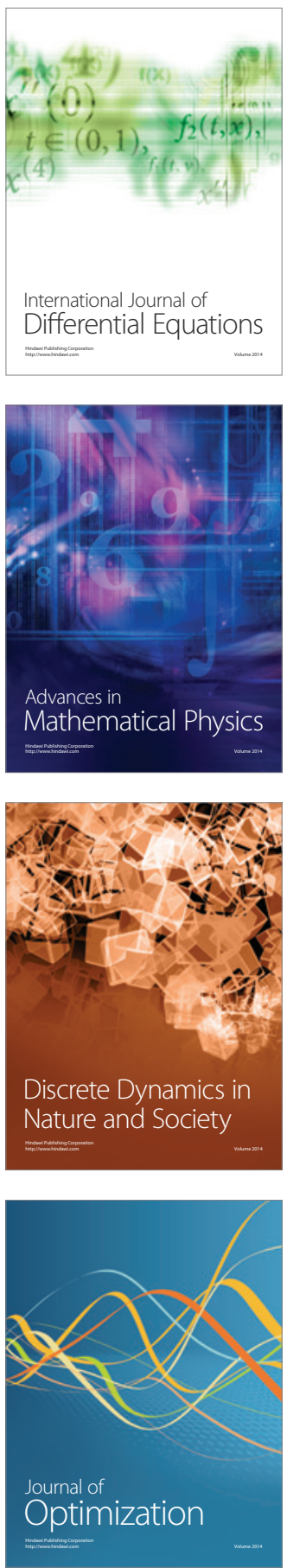